\title{
AUTHOR INDEX TO VOLUME 52
}

Author

AzIz, A. K. \&

MONK, PETER

BABUŠKA, I. \& OSBORN,

J. E.

BACH, ERIC \&

SHALLIT, JEFFREY

BALES, LAURENCE A. \&

Dougalis, Vassilios A.

BAYLISS, ALVIN,

LI, YANYAN \&

Morawetz, CathleEN S.

BELLEN, A.,

JACKIEWICZ, Z.,

VERMIGLIO, R. \&

ZENNARO, M.

BRAMBLE, JAMES H.,

PASCIAK, JOSEPH E.,

SAMMON, PETER H. \&

THOMÉE, VIDAR

BRENNER, SUSANNE C.

BROWNING, G. L. \&

KREISS, H.-O.

BUCHMANN, JOHANNES \&

FORD, DAVID

BUCHMANN, JOHANNES \&

Pethö, Attila

CAI, WEI,

GOTTLIEB, DAVID \&

SHU, CHI-WANG

COCKBURN, BERNARDO \&

ShU, CHI-WANG

COMOdI, M. I.

Creutzburg, R. \&

TASCHE, $M$.

Crouzeix, Michel \&

FALK, RICHARD S.

Del prete, Vincenza

Dougalis, Vassilios A.

DOUGHERTY, RANDALL L., EDELMAN, ALAN \& HYMAN, JAMES M.

DOUGLAS, JIM JR. \&

WANG, JUNPING

Edelman, Alan
Title

Page

Continuous Finite Elements in Space and Time for the Heat Equation . . . . . . . . . . . . 255

Finite Element-Galerkin Approximation of the Eigenvalues and Eigenvectors of Selfadjoint Problems . . . . . 275

Factoring with Cyclotomic Polynomials . . . . . . . 201

Cosine Methods for Nonlinear Second-Order Hyperbolic Equations . . . . . . . . . . . . . . . 299

Scattering by a Potential Using Hyperbolic Methods . . . 321

Natural Continuous Extensions of Runge-Kutta Methods for Volterra Integral Equations of the Second Kind and Their Applications . . . . . . . . . . . . . . .

Incomplete Iterations in Multistep Backward Difference Methods for Parabolic Problems with Smooth and Nonsmooth Data ......................

An Optimal-Order Multigrid Method for P1 Nonconforming Finite Elements . . . . . . . . . . . .

Comparison of Numerical Methods for the Calculation of Two-Dimensional Turbulence . . . . . . . . . . . . .

On the Computation of Totally Real Quartic Fields of Small Discriminant. . . . . . . . . . . . . . . . .

Computation of Independent Units in Number Fields by Dirichlet's Method . . . . . . . . . . . . . . . . .

Essentially Nonoscillatory Spectral Fourier Methods for Shock Wave Calculations . . . . . . . . . . . . . .

TVB Runge-Kutta Local Projection Discontinuous Galerkin Finite Element Method for Conservation Laws II: General Framework . . . . . . . . . . . . . . . . .

The Hellan-Herrmann-Johnson Method: Some New Error Estimates and Postprocessing . . . . . . . . . .

Parameter Determination for Complex Number-Theoretic Transforms Using Cyclotomic Polynomials . . . . . . .

Nonconforming Finite Elements for the Stokes Problem .

Quadratic Convergence of Vortex Methods . . . . . . . . See: Bales, laurence A. \& Dougalis, Vassilios A.

Nonnegativity-, Monotonicity-, or Convexity-Preserving Cubic and Quintic Hermite Interpolation . . . . . . .

An Absolutely Stabilized Finite Element Method for the Stokes Problem . . . . . . . . . . . .

See: Dougherty, Randall L., Edelman, Alan \& HYMAN, JAMES M. . . . . . . . . . 5 5

9 
Author

Title

Page

EICHENAUER-HERRMANN, JÜRGEN,

GROTHE, HOLGER \&

LEHN, JÜRGEN

ENGQUIST, BJÖRN,

LÖTSTEDT, PER \&

SJÖGREEN, BJÖRN

FALK, RICHARD S.

FORD, DAVID

GARABEDIAN, P. R.

Gelman, Alex \&

RHEINBOLDT, WERNER C.

GORDON, DANIEL M.

GOTTLIEB, DAVID

GROTHE, HOLGER

HYMAN, JAMES M.

ISAACSON, DAVID \&

ISAACSON, ELI L.

ISAACSON, ELI L.

JACKIEWICZ, Z.

KEELING, STEPhEN L.

KREISS, H.-O.

LEHN, JÜRGEN

LI, YANYAN

LIE, IVAR \&

NøRSETT, SYVERT P.

LYNESS, JAMES N.

LÖTSTEDT, PER

MASTRoianni, GIUSEPPE

MONK, PETER

MORAIN, FRANÇOIS

Morawetz, Cathleen S.

MOSTREL, MARCO

MOSCHÉ

NIEDERREITER, HARALD

NøRSETT, SYVERT P.

On the Period Length of Pseudorandom Vector Sequences Generated by Matrix Generators . . . . . . . . . .

Nonlinear Filters for Efficient Shock Computation . . . .

See: Crouzeix, Michel \& Falk, Richard S. . . . .

See: Buchmann, Johannes \& Ford, David . . . .

161

Design of a Large Helical System for Magnetic Fusion Experiments . . . . . . . . . . . . .

On the Computation of Finite Invariant Sets of Mappings

On the Number of Elliptic Pseudoprimes . . . . . . . .

231

See: Cai, Wei, Gottlieb, David \& Shu, Chi-Wang

See: Eichenauer-Herrmann, Jürgen, Grothe, HOLGER \& LEHN, JÜRGEN . . . . . . . . . . .

See: Dougherty, Randall L., Edelman, Alan \& HYMAN, JAMES M. . . . . . . . . . . .

Comment on Calderón's Paper: "On an Inverse Boundary Value Problem" ... . . . . . . . . . . . . 553

See: ISAACSON, DAVID \& ISAACSON, ELI L. . . . . . . 553

See: Bellen, A., Jackiewicz, Z., Vermiglio, R. \&

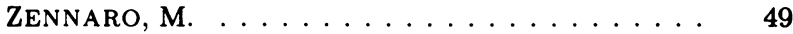

Galerkin/Runge-Kutta Discretizations for Parabolic Equations with Time-Dependent Coefficients . . . . . . .

See: Browning, G. L. \& Kreiss, H.-O. . . . . . . .

See: Eichenauer-HerRmanN, JÜrgen, Grothe, HOLGER \& LEHN, JÜRGEN . . . . . . . . . . . .

See: Bayliss, Alvin, Li, Yanyan \& Morawetz, CATHLEEN S. . . . . . . . . . . . . . . . . 321

Superconvergence for Multistep Collocation $\ldots \ldots \ldots \quad 65$

See: Sloan, IAN H. \& Lyness, James N. . . . . . . 81

See: ENGquist, BJÖRN, LÖTSTEDT, PER \& SJÖgREeN, BJÖRN . . . . . . . . . . . . . .

On the Convegence of Product Formulas for the Evaluation of Certain Two-Dimensional Cauchy Principal Value Integrals . . . . . . . . . . . . . . . 95

See: Aziz, A. K. \& Monk, Peter . . . . . . . 255

On the $\mathrm{lcm}$ of the Differences of Eight Primes . . . . . 225

See: Bayliss, Alvin, Li, Yanyan \& Morawetz, CathleEn S. . . . . . . . . . . . . . 321

On Some Numerical Schemes for Transonic Flow Problems 587

The Serial Test for Congruential Pseudorandom Numbers Generated by Inversions . . . . . . . . . . . . . 135

See: Lie, IVAR \& NøRSETt, SyVERT P. . . . . . . . $\quad 65$

OSBORN, J. E.

PASCIAK, JOSEPH E.

See: BABUŠKA, I. \& OSBORN, J. E. . . . . . . . . . .

See: BRAmble, James H., PASciaK, Joseph E., Sammon, Peter H. \& Thomée, Vidar . . . . . . 
Author

Title

Page

Pethö, Attila

See: Buchmann, Johannes \& Pethö, Attila . . .

POTLER, AARON

PUCKETT, ElbRIDGE

GERRY

See: Young, JefF \& Potler, AARon . . . . . . . .

Convergence of a Random Particle Method to Solutions of the Kolmogorov Equation $u_{t}=\nu u_{x x}+u(1-u) \ldots \quad 615$

Rheinboldt, Werner C. See: Gelman, Alex \& Rheinboldt, Werner C. . .

ROBERTS, STEPHEN

SACCHI LANDRIANI, GIOVANNI \& VANDEVEN, HERVÉ

SAMMON, PETER H.

SHALLIT, JEFFREY

Convergence of a Random Walk Method for the Burgers Equation . . . . . . . . . . . .

Polynomial Approximation of Divergence-Free Functions 221 545 647 103

SHU, CHI-WANG

SHU, CHI-WANG

SJÖGREEN, BJÖRN

SLOAN, IAN H. \& LYNESS, JAMES N.

SMITH, DAVID M.

STEPHAN, E. P. \&

SURI, M.

STROEKER, R. J.

SURI, M.

SWARTZ, BLAIR

TASCHE, M.

THOMÉE, VIDAR

VANDEVEN, HERVÉ

VERMIGLIO, R.

WANG, JUNPING

YOUNG, JEFF \&

POTLER, AARON

ZENNARO, M.

See: Bramble, James H., Pasciak, Joseph E., SAMMON, PETER H. \& THOMÉE, Vidar . . . . . . 339

See: BACH, ERIC \& SHALLIT, JefFrEY . . . . . . . 201

See: Cai, Wei, Gottlieb, David \& Shu, Chi-Wang 389

See: Cockburn, Bernardo \& ShU, ChI-WANG . . 411

$S e e$ : ENGQUIST, BJÖRN, LÖTSTEDT, PER \& SJÖGREEN, BJÖRN . . . . . . . . . . . . . . . 509

The Representation of Lattice Quadrature Rules as Multiple Sums . . . . . . . . . . . . . . . 81

Efficient Multiple-Precision Evaluation of Elementary Functions. . . . . . . . . . . . . . . 131

On the Convergence of the $p$-Version of the Boundary Element Galerkin Method .............. 31

On Quartic Thue Equations with Trivial Solutions . . . 175

See: STEPHAN, E. P. \& SURI, M. . . . . . . . . 31

The Second-Order Sharpening of Blurred Smooth Borders 675

See: Creutzburg, R. \& Tasche, M. . . . . . . . . 189

See: Bramble, James H., Pasciak, Joseph E., SAMmon, Peter H. \& ThOMÉE, Vidar . . . . . 339

See: SACCHI Landriani, GIOVANni \& VANDEVEn,

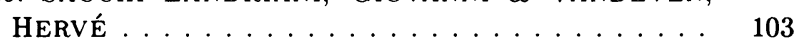

See: Bellen, A., Jackiewicz, Z., Vermiglio, R. \&

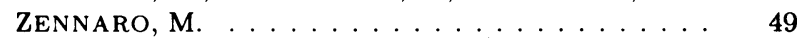

See: Douglas, Jim JR. \& WANG, JUNPING . . . . . 495

First Occurrence Prime Gaps . . . . . . . . . . 221

See: Bellen, A., Jackiewicz, Z., Vermiglio, R. \& ZENNARO, M. 